\title{
Reproducibility of serum cytokines in an elderly population
}

\author{
Jing Guo', Nicole Schupf ${ }^{1,2,3,4}$, Richard P. Mayeux $x^{1,2,3,4}$ and Yian Gu ${ }^{1,2,3,4^{*}}$ (B)
}

\begin{abstract}
Background: It is important to assess the temporal reproducibility of circulating cytokines for their utility in epidemiological studies. However, existing evidence is limited and inconsistent, especially for the elderly population.

Methods: Sixty-five elderly (mean age $=77.89 \pm 6.14$ years) subjects were randomly selected from an existing prospective cohort study. Levels of 41 cytokines in 195 serum samples, collected at three separate visits that were up to 15.26 years apart, were measured by the Luminex technology. The temporal reproducibility of cytokines was estimated by the intraclass correlation coefficient (ICC) calculated using a mixed-effects model. In addition, data analyses were stratified by the median (4.49 years) of time intervals across sample collection. Sensitivity analyses were performed when excluding subjects with undetectable samples.
\end{abstract}

Results: A total of 23 cytokines were detectable in more than $60 \%$ of samples. Fair to good (ICC $=0.40$ to 0.75 ) and excellent (ICC > 0.75) reproducibility was found in 10 (Eotaxin, VEGF, FGF-2, G-CSF, MDC, GM-CSF, TGFa, IP-10, MIP$1 \beta$, IL-1RA) and 5 (GRO, IFNY, IL-17, PDGF-AA, IL-4) cytokines, respectively. The results were not changed dramatically in the stratification and sensitivity analyses.

Conclusions: Serum levels of the selected 15 cytokines measured with Luminex technology displayed fair to excellent within-person temporal reproducibility among elderly population.

Keywords: Reproducibility, Serum, Cytokines, Elderly population

\section{Background}

Cytokines are secreted polypeptides or glycoproteins that regulate proliferation, apoptosis, and angiogenesis, and involved in the development and progression of various diseases [1]. Cytokine-mediated intercellular communication is regarded as the main mechanism of crosstalk between immune cells [2]. Cytokine and other inflammatory biomarkers play important roles in the pathophysiology of chronic inflammatory diseases [3], cardiovascular diseases [4], neuroinflammation [5], asthma [6], and cancer [7].

\footnotetext{
* Correspondence: yg2121@cumc.columbia.edu

${ }^{1}$ Taub Institute for Research in Alzheimer's Disease and the Aging Brain, Columbia University, New York, NY, USA

2Department of Neurology, Columbia University, New York, NY, USA

Full list of author information is available at the end of the article
}

Circulating inflammatory molecules are widely used in epidemiological studies to investigate their association with chronic diseases [8-10]. In some population-based studies, especially the large-scale cohort studies with a long follow-up period, a single measure of blood sample collected at baseline is usually employed to represent the long-term state of inflammation of participants. However, high intra-individual variability of cytokines may lead to a misclassification of inflammatory status and biased association analyses. Therefore, it is important to establish the temporal reproducibility of cytokines when investigating the cytokine-disease associations [11].

Several studies have explored the intra-individual temporal reproducibility of cytokines in humans (see Additional file 1: Table S1). However, there were some limitations in the previous studies, including small sample size [12-16], assessment of short-term variability 
[14, 17-20], analysis of a limited number of cytokines $[15,16,18,21]$, or limited to specific populations such as women [11] or with particular physiological status of pregnancy [19]. Furthermore, the temporal reproducibility of cytokines has been predominantly examined for young and middle-aged adults, and the average age of participants was less than 65 years in many previous studies (see Additional file 1: Table S1). As cytokine levels tend to increase with advanced age [18], a phenomenon called inflammageing [22, 23], it is important to evaluate the temporal reproducibility of cytokines in elderly populations.

In the present study, we aimed to investigate the detectability and temporal reproducibility of a large panel of inflammatory biomarkers among 65 elderly $(\geq 65$ years) subjects, with 3 serum samples each. Results from this study will be useful to select appropriate cytokines as inflammatory biomarkers for elderly population in the future epidemiological studies.

\section{Methods}

\section{Study design and participants}

The Washington Heights-Inwood Community Aging Project (WHICAP) is an ongoing, prospective, population-based cohort study which is conducted to identify risk factors and biomarkers for aging and dementia. The sampling strategies, recruitment and examination methodology of WHICAP have been described previously $[24,25]$. Participants in WHICAP were socioeconomically and racially diverse, community residents in northern Manhattan, aged $\geq 65$ years, and fluent in English or Spanish. At baseline and at the follow-up visits every $18-24$ months, participants received comprehensive medical, physical, neurological and neuropsychological examinations. Blood samples were collected at the enrollment and the follow-up visits.

For the current study, we selected a total of 65 participants from the second and third waves of WHICAP who met the following criteria: (a) $\geq 3$ blood samples donated at different visits, (b) large number of aliquots left, (c) without history of diagnosed dementia, and (d) complete information on ages at blood draw, sex, race/ethnicity and years of education. To ensure individuals with certain demographic characteristics to be included in this sample, we randomly selected subjects from each of the 24 strata, defined by sex (male vs. female), race/ethnicity (White, Black, Hispanic), waves (1999 vs. 2009), and age groups (< 80 vs. $\geq 80$ years). For each selected individual, we selected one serum sample from the baseline, the most recent, and a middle visit. With three repeated samples each for a total of 65 subjects, we had $80 \%$ power to detect acceptable ICC of $\geq 0.55$ with narrow $95 \%$ confidence interval $(\mathrm{CI})(< \pm 0.13)$, or to detect acceptable ICC of $\geq 0.40$ with narrow $95 \%$ CI $(< \pm 0.15)$.

\section{Cytokine quantification}

Peripheral venous blood samples were collected from the participants at the time of their health examination, and were stored at $-80^{\circ} \mathrm{C}$. Never-thawed serum samples were packed in dry-ice and sent to the laboratory for analysis. A panel of 41 inflammatory biomarkers were selected according to the results of previous literatures (see Additional file 1: Table S1), biological functions of cytokines, and availability of commercial assay kits, and were analyzed by the Luminex technology. The magnetic bead-based sandwich immunoassays for cytokines using the MILLIPLEX Human Cytokine Panel 1 (HCYTOMAG-60 k) (MilliporeSigma, St. Louis, MO) were performed according to the manufacturer's instructions. The serum samples $(25 \mu \mathrm{L})$ were analyzed in duplicate wells using a Luminex 200 (Luminex Corp, Austin, TX). The cytokine concentrations were determined by Luminex xPONENT v4.2 and MILLIPLEX Analyst v5.1 using 5-p $\log$ analysis. All assays were performed using the same lot of Luminex reagents. All samples from the same subject are always in the sample plate. Two sets of quality controls spiked in serum, provided by the manufacturer, were run in duplicate across the plates. This is an ideal substitute for the pool samples since the sample type in this study was also serum. The inter-assay precision is excellent with a mean coefficient of variation (CV) of $6.74 \%$ (range: 1.87 to $16.03 \%$ ), well below the $20 \%$ accepted cut off for a ligand-binding assay suggested by the Bioanalytical Method Validation Guidelines for Industry in 2018 [26].

Samples with cytokine levels less than the lower limit of detection (LLOD) were assigned a value of LLOD divided by the square root of two [19]. Observations beyond the upper limit of detection (ULOD) were substituted with ULOD for data analysis. Samples with values between the LLOD and ULOD were regarded as detectable for specific cytokines. The cytokines were retained for further analyses when the proportions of detectable values were at least $60 \%$ among all the 195 blood samples [11].

\section{Statistical analysis}

Due to the skewed distribution, cytokine levels were natural logarithm (log)-transformed for approximately normal distribution. The characteristics of subjects were presented as mean [standard deviance (SD)] and number [proportion (\%)] for continuous and categorical variables, respectively. The repeated measures correlation $(95 \% \mathrm{CI})$ between different cytokines were estimated with the $\mathrm{R}$ package "rmcorr" which accounts for non-independence among measurements from the same subject [27].

The intraclass correlation coefficient (ICC) was employed to quantify the temporal reproducibility of cytokines. The mixed-effects model, fitted by the $\mathrm{R}$ 
package "lme4" (version 1.1.21) [28], was established when using the levels of each cytokine as dependent variable, setting the random intercept on the repeated measures, and adjusting for the covariates of age at recruitment (years), sex (male, female), race/ethnicity (non-Hispanic White, non-Hispanic Black, Hispanic) and time intervals across blood collection (years). Based on the results of mixed-effects model, ICC was calculated by dividing the between subject variance by the total variance [29]. The model-based parametric bootstrap for mixed models was used to estimate the $95 \%$ CI of ICC. For the main analyses, ICC was calculated using all three repeated measurements. The ICC levels of < $0.40,0.40 \sim 0.75$ and $>0.75$ mean poor, fair to good and excellent reproducibility, respectively [30].

To examine the impact of time intervals on the cytokine reproducibility, data analyses were performed by the stratification of median of time intervals between the 1st and 3rd measures. Besides, we also repeated analyses by excluding samples with undetected values $(<$ LLOD or > ULOD), or by additionally adjusting for the baseline comorbidities including hypertension, diabetes and cardiovascular diseases, or by additionally adjusting for the body mass index (BMI, $\mathrm{kg} / \mathrm{m}^{2}$ ) categories (normal weight $[<25]$, overweight [ $\geq 25$ and $<30$ ], and obese [> 30]) at each visit, or by additionally adjusting for the time of day (ante meridiem or post meridiem) of blood sample collection. Sensitivity analyses were also conducted by the stratification of sex.

All the data analyses were performed with $\mathrm{R}$ (version 3.6.1).

\section{Results}

\section{Characteristics of subjects}

Participant characteristics are presented in Table 1. Among the 65 subjects, the mean of age at the collection of first blood sample was 77.89 ( $\mathrm{SD}=6.14$; range $=67.34$ to 90.49 ) years. By design, about half of the subjects were males, about two thirds were $<80$ years and the rest $\geq 80$ years, the White, Black and Hispanic population each accounted for nearly one third of the sample, and the proportions of individuals from the second and third waves of WHICAP were 55.38 and $44.62 \%$, respectively. The average (SD) years of education was 12.26 (4.95). At the collection of first blood sample, subjects at BMI categories of normal weight, overweight, and obese accounted for 26.42, 35.85, and $37.74 \%$, respectively. More than half (58.00\%) of the first blood samples were collected in the morning. Participants with hypertension, diabetes, and cardiovascular diseases at baseline accounted for 92.31, 29.23 , and $56.92 \%$, respectively. The medians of time intervals were 2.45 [interquartile range $(\mathrm{IQR})=2.00$ to 5.72 ], $1.78(\mathrm{IQR}=1.63$ to 2.19$)$, and $4.49(\mathrm{IQR}=3.91$ to 8.09$)$ years between the 1st and 2nd, between the 2nd and 3rd, and between the 1st and 3rd blood samples, respectively.

\section{Levels of cytokines}

The intra- and inter-assay CV, proportion of detectable samples, and median and range of cytokines were estimated among the 195 specimens from 65 participants (Table 2). The detectable percentage ranged from 4.62 to $100 \%$ for the 41 cytokines, of which 23 analytes with detectable percentage $\geq 60 \%$ were retained for further assessment on the temporal reproducibility. The results of correlations indicated that some cytokines were significantly correlated with each other $(p<0.05)$ (see Additional file 1: Fig. S1).

\section{Temporal reproducibility of cytokines}

The ICC (95\% CI) of cytokines measured in all samples collected at three separate visits was ranked and presented in Fig. 1. The lowest ICC (95\% CI) was approximated to zero for IL8, and the highest was 0.87 (0.81, $0.92)$ for GRO. Results showed that the temporal reproducibility was fair to good for 10 cytokines (ICC $=0.40$ 0.75; including Eotaxin, VEGF, FGF-2, G-CSF, MDC, GM-CSF, TGF $\alpha$, IP-10, MIP-1 $\beta$, IL-1RA), and were excellent for 5 cytokines (ICC > 0.75; including GRO, IFN $\gamma$, IL-17, PDGF-AA, IL-4). The remaining 8 cytokines displayed poor temporal reproducibility.

Stratification analyses were additionally conducted, dividing by the median ( 4.49 years) of time intervals between the 1st and 3rd blood samples (Fig. 2). Compared with the results of the main analyses using all the subjects, the ICCs remained similar except for TNF $\alpha$, for which the ICC increased into fair to good levels among those with time intervals $\geq 4.49$ years.

After excluding the subjects with undetectable levels of cytokines, for the cytokines with detectable rates less than $100 \%$ (Table 2), the results remained similar although in general the ICC reduced slightly except for MCP-3, which the ICC increased $(0.41,95 \% \mathrm{CI}=0.20$ to 0.62) (see Additional file 1: Fig. S2). Robust results were obtained when additionally adjusting for the baseline comorbidities (see Additional file 1: Fig. S3), or for BMI categories (see Additional file 1: Fig. S4), or for the time of day of blood sample collection (see Additional file 1: Fig. S5), except for IP-10 and IL-1RA of which the ICC decreased when adjusting for BMI, and except for TNFo of which ICC increased when adjusting for the time of day of blood sample collection. The ICC levels stratified by sex were similar compared with the main results of all subjects, except that the ICC of TNF $\alpha$ increased into fair to good levels in males (see Additional file 1: Fig. S6).

\section{Discussion}

In the present study of 65 elderly subjects from WHICAP study, we assessed the temporal reproducibility of 
Table 1 Characteristics of participants across three repeated measurements

\begin{tabular}{|c|c|c|c|}
\hline Characteristics & The 1st measurement & The 2nd measurement & The 3rd measurement \\
\hline Sample size & 65 & 65 & 65 \\
\hline Age (years), mean (SD) & $77.89(6.14)$ & $82.50(4.85)$ & $84.43(4.85)$ \\
\hline $65-75, n(\%)$ & $23(35.38 \%)$ & $5(7.69)$ & $2(3.08)$ \\
\hline $75-80, n(\%)$ & $19(29.23 \%)$ & $13(20.00)$ & $9(13.85)$ \\
\hline$\geq 80, \mathrm{n}(\%)$ & $23(35.38 \%)$ & $47(72.31)$ & $54(83.08)$ \\
\hline \multicolumn{4}{|l|}{ Gender, n (\%) } \\
\hline Male & $32(49.23)$ & $32(49.23)$ & $32(49.23)$ \\
\hline Female & $33(50.77)$ & $33(50.77)$ & $33(50.77)$ \\
\hline \multicolumn{4}{|l|}{ Race/ethnicity, n (\%) } \\
\hline White & $22(33.85)$ & $22(33.85)$ & $22(33.85)$ \\
\hline Black & $21(32.31)$ & $21(32.31)$ & $21(32.31)$ \\
\hline Hispanic & $22(33.85)$ & $22(33.85)$ & $22(33.85)$ \\
\hline Education duration (years), mean (SD) & $12.26(4.95)$ & $12.26(4.95)$ & $12.26(4.95)$ \\
\hline \multicolumn{4}{|l|}{ BMI categories, $\mathrm{n}(\%)^{\text {a }}$} \\
\hline Normal & $14(26.42)$ & $14(25.45)$ & $16(29.09)$ \\
\hline Overweight & $19(35.85)$ & $20(36.36)$ & $22(40.00)$ \\
\hline Obese & $20(37.74)$ & $21(38.18)$ & $17(30.91)$ \\
\hline \multicolumn{4}{|l|}{ Time of day of blood collection, $n(\%)^{a}$} \\
\hline Ante meridiem & $29(58.00)$ & $37(57.81)$ & $43(66.15)$ \\
\hline Post meridiem & $21(42.00)$ & $27(42.19)$ & $22(33.85)$ \\
\hline \multicolumn{4}{|l|}{ WHICAP wave, n (\%) } \\
\hline The second wave (1999 cohort) & $36(55.38)$ & $36(55.38)$ & $36(55.38)$ \\
\hline The third wave (2009 cohort) & $29(44.62)$ & $29(44.62)$ & $29(44.62)$ \\
\hline \multicolumn{4}{|l|}{ Baseline hypertension, n (\%) } \\
\hline No & $5(7.69)$ & - & - \\
\hline Yes & $60(92.31)$ & - & - \\
\hline \multicolumn{4}{|l|}{ Baseline diabetes, n (\%) } \\
\hline No & $46(70.77)$ & - & - \\
\hline Yes & $19(29.23)$ & - & - \\
\hline \multicolumn{4}{|l|}{ Baseline cardiovascular diseases, n (\%) } \\
\hline No & $28(43.08)$ & - & - \\
\hline Yes & $37(56.92)$ & - & - \\
\hline
\end{tabular}

SD standard deviance, $n$ number of subjects, \% proportion, BMI body mass index, WHICAP Washington Heights-Inwood Community Aging Project.

${ }^{a}$ Sum of frequency of some characteristics was less than 65 at specific measurement due to missing data

serum cytokines in samples collected at three separate visits over a relatively long period of time. The results revealed that 15 out of 41 cytokines measured by the Luminex technology were detectable in over $60 \%$ of serum samples and had good reproducibility (ICC > 0.40 ), indicating that a single measurement of these biomarkers well captures the within-individual average level over a long period of time.

By definition, ICC measures the inter-person variability relative to the total variability [29]. The high values of ICC indicated that the variation of cytokine levels was considerably higher between subjects than within subjects, which supports the good reproducibility of these biomarkers over a long term. Therefore, cytokines having high ICC can be used in the epidemiological studies focusing on the associations between one-time measured cytokines and diseases.

The temporal reproducibility of circulating cytokines measured by multiplex immunoassays or ELISA has been reported previously. Our findings are consistent with previous studies, supporting good to excellent ICC for IL-17 [17], Eotaxin [11], G-CSF [12, 14, 17], MIP-1 $\beta$ $[14,17]$ and IL-1RA [11]. However, inconsistencies also exist, which might be due to the discrepancies in study 
Table 2 Levels of serum cytokines

\begin{tabular}{|c|c|c|c|c|c|}
\hline Cytokines & Intra-assay CV (\%) & Inter-assay CV (\%) & Detectable proportions (\%) ${ }^{a}$ & Median $\left(\mathrm{P}_{25}-\mathrm{P}_{75}\right)(\mathrm{pg} / \mathrm{mL})$ & Range $(\mathrm{pg} / \mathrm{mL})$ \\
\hline$\overline{E G F}$ & 0.91 & 7.19 & 99.49 & $226.71(126.59,374.77)$ & $<3.17$ to 1657 \\
\hline Eotaxin & 1.37 & 3.48 & 100 & $134.79(98.66,183)$ & 8.46 to 562.15 \\
\hline FGF-2 & 1.68 & 7.56 & 88.72 & $48.23(23.39,105.09)$ & $<13.05$ to 429.02 \\
\hline Flt-3 L & 3.17 & 7.99 & 48.21 & $3.29(3.29,30.1)$ & $<4.65$ to 1769 \\
\hline Fractalkine & 2.05 & 12.69 & 43.59 & $21.48(18.72,61.34)$ & $<26.48$ to $>9834$ \\
\hline G-CSF & 3.06 & 8.69 & 91.28 & $20.45(11.17,49.02)$ & $<3.97$ to 404.65 \\
\hline GM-CSF & 7.45 & 6.89 & 80.51 & $7.58(3.54,17.48)$ & $<3.16$ to 1287 \\
\hline GRO & 1.07 & 4.65 & 77.44 & $2216(1264,7288.5)$ & 470.26 to $>9906$ \\
\hline IFNa2 & 5.34 & 5.92 & 55.90 & $7.9(3.23,25.39)$ & $<4.57$ to 924.48 \\
\hline IFNY & 0.44 & 8.50 & 75.38 & $5.06(2.07,11.76)$ & $<2.04$ to 1183 \\
\hline$\| \mathrm{L}-10$ & 5.26 & 4.49 & 25.13 & $2.21(2.21,3.21)$ & $<3.13$ to 1304 \\
\hline IL-12p40 & 3.47 & 6.37 & 30.26 & $4.79(4.79,11.68)$ & $<6.78$ to 605.62 \\
\hline IL-12p70 & 0.99 & 2.68 & 27.69 & $2.02(2.02,3.74)$ & $<2.85$ to 655.57 \\
\hline IL-13 & 3.05 & 5.86 & 26.15 & $3.33(3.33,6.25)$ & $<4.71$ to 598.37 \\
\hline IL-15 & 4.94 & 6.30 & 34.36 & $2.04(2.04,4.3)$ & $<2.88$ to 153.79 \\
\hline IL-17 & 2.90 & 4.36 & 70.77 & $2.57(0.73,7.72)$ & $<1.03$ to 451.19 \\
\hline IL-1a & 9.92 & 5.47 & 43.59 & $3.6(3.6,25.14)$ & $<5.09$ to 3539 \\
\hline $\mathbb{I L}-1 \beta$ & 5.15 & 8.15 & 53.33 & $1.48(0.88,4.86)$ & $<1.24$ to 2581 \\
\hline IL-1RA & 3.02 & 8.20 & 90.26 & $15.7(7.66,31.01)$ & $<3.65$ to 3857 \\
\hline IL-2 & 3.14 & 4.48 & 28.72 & $1.98(1.98,3.62)$ & $<2.80$ to 86.71 \\
\hline IL-3 & 11.71 & 12.30 & 4.62 & $1.97(1.97,1.97)$ & $<2.78$ to 13.07 \\
\hline IL-4 & 8.57 & 7.22 & 83.51 & $31.18(9.96,79.75)$ & $<5.42$ to 375.29 \\
\hline IL-5 & 4.03 & 1.87 & 22.05 & $0.76(0.76,0.94)$ & $<1.07$ to 72.28 \\
\hline IL-6 & 4.99 & 6.19 & 42.56 & $1.32(1.32,19.72)$ & $<1.86$ to 7220 \\
\hline IL-7 & 5.37 & 4.70 & 43.59 & $2.64(2.34,7.73)$ & $<3.31$ to 119.38 \\
\hline IL-8 & 2.91 & 4.22 & 97.44 & $36.4(15.12,245.41)$ & 3.84 to $>9613$ \\
\hline IL-9 & 3.91 & 5.98 & 15.38 & $1.99(1.99,1.99)$ & $<2.81$ to 128.69 \\
\hline $\mid \mathrm{P}-10$ & 2.69 & 3.44 & 100 & $222.35(175.6,307.84)$ & 49.15 to 1009 \\
\hline MCP-1 & 3.53 & 5.93 & 100 & $590.84(429.88,902.12)$ & 118.05 to 9309 \\
\hline MCP-3 & 0.72 & 5.50 & 61.54 & $6.17(2.47,34.91)$ & $<3.50$ to 2891 \\
\hline MDC & 9.14 & 8.11 & 100 & $1043(758.06,1409)$ & 234.05 to 6725 \\
\hline MIP-1a & 8.53 & 5.06 & 88.21 & $19.24(6.44,69.77)$ & $<3.12$ to $>5472$ \\
\hline MIP-1 $\beta$ & 0.65 & 6.53 & 99.49 & $48.04(28,89.12)$ & $<4.50$ to 1251 \\
\hline PDGF-AA & 2.00 & 3.91 & 99.49 & $3576(2533.5,4803.5)$ & 305.27 to $>8786$ \\
\hline PDGF-AB/BB & 6.91 & 9.83 & 30.77 & $9490(8841,9490)$ & 3148 to $>9490$ \\
\hline RANTES & 8.08 & 12.69 & 96.92 & $2730(1845.5,3724.5)$ & 619.28 to $>13,438$ \\
\hline sCD40L & 1.74 & 6.59 & 84.10 & $9529(4893.5,13,789)$ & $<2.86$ to $>17,067$ \\
\hline TGFa & 1.09 & 16.03 & 66.15 & $4.72(2.18,8.31)$ & $<3.09$ to $>5120$ \\
\hline TNFa & 2.78 & 7.59 & 95.90 & $16.48(9.66,27.52)$ & $<2.93$ to 716.76 \\
\hline TNF $\beta$ & 4.15 & 5.78 & 19.49 & $2.01(2.01,2.01)$ & $<2.84$ to 639.95 \\
\hline VEGF & 4.22 & 7.00 & 88.21 & $161.45(71.86,326.21)$ & $<20.50$ to 919.48 \\
\hline
\end{tabular}

$C V$ coefficient of variation, $P_{25}-P_{75}$ the 25th to the 75th percentiles, EGF epidermal growth factor, FGF-2 fibroblast growth factor 2, Flt-3 L fms-like tyrosine kinase 3 ligand, G-CSF granulocyte colony-stimulating factor, GM-CSF granulocyte macrophage colony-stimulating factor, GRO growth regulated oncogene, IFNa2 interferon alpha 2, IFNY interferon gamma, IL interleukin, IL-1RA interleukin-1 receptor antagonist, IP-10 interferon gamma-induced protein 10, MCP monocyte chemotactic protein, MDC macrophage-derived chemokine, MIP macrophage inflammatory proteins, PDGF platelet-derived growth factor, RANTES regulated on activation, normal T cell expressed and secreted, TGF transforming growth factor, TNF tumor necrosis factor, VEGF vascular endothelial growth factor, $S C D 40 L$ soluble cluster of differentiation 14 ligand.

a Values in bold mean that the proportions of subjects with detectable values (between LLOD and ULOD) were $<60 \%$. A total of 18 cytokines were excluded in the further data analyses due to the low detectable rate 


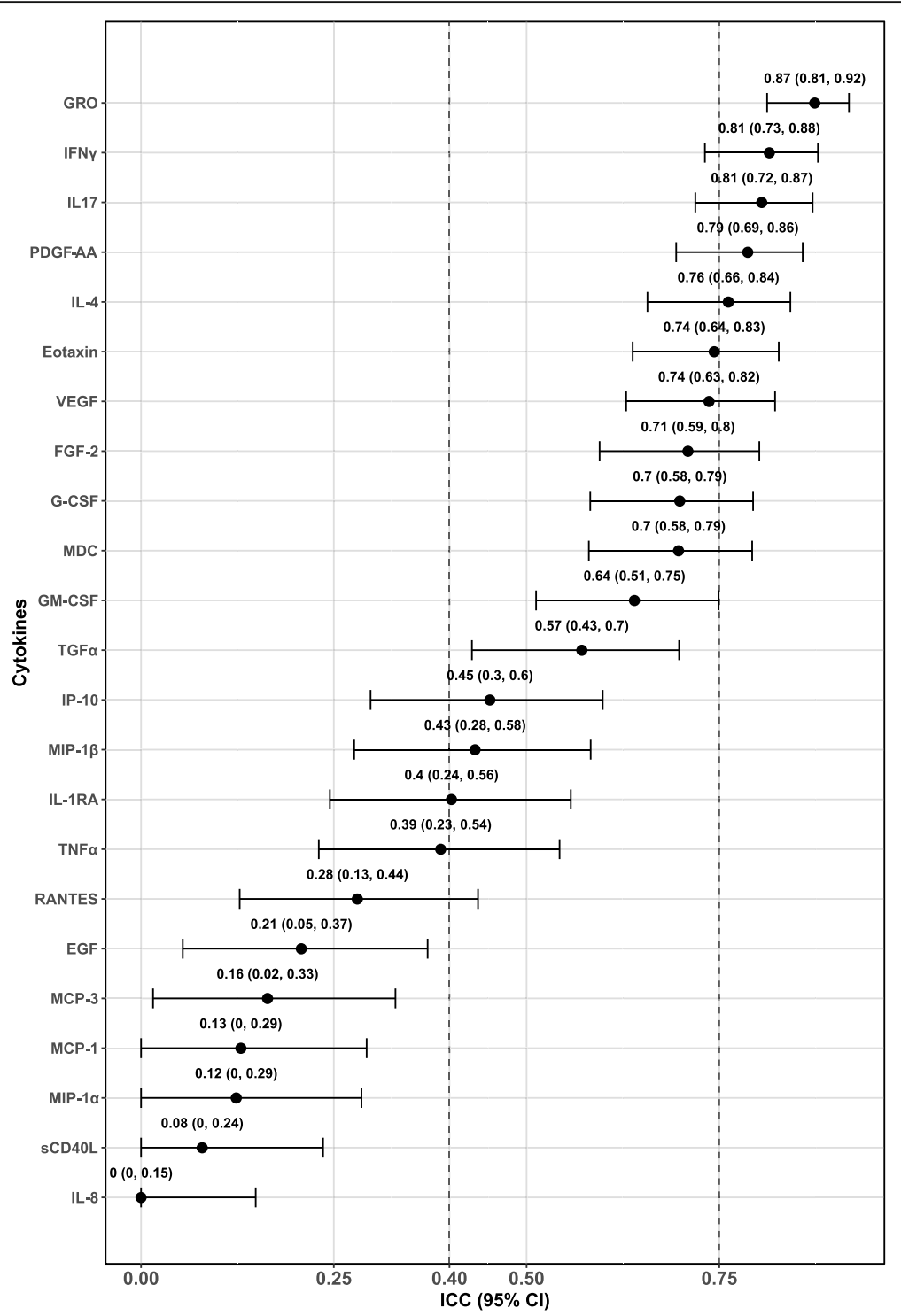

Fig. 1 ICC (95\% Cl) of cytokines. The dots and horizontal bars represent the ICC and corresponding confidence intervals, respectively. ICC was estimated by the mixed-effects model adjusting for age at recruitment, gender, race/ethnicity and time spans across blood collection. The ICC levels of $0.40 \sim 0.75$ and $>0.75$ are corresponding to fair to good and strong reliabilities, respectively. ICC: intraclass correlation coefficient; Cl: confidence interval

design, sample size, type of specimen, and assay methods $[18,31]$. For example, extremely low ICC of IL-8 was found in our study (near to 0 ) and other studies (from 0.02 to 0.05$)[11,14]$ in which cytokine levels were examined in serum. Conversely, a good reproducibility of IL-8 has been demonstrated in plasma $(>0.40)[12,14$, 18]. It has been reported that the concentrations of cytokines and the corresponding ICC are higher in plasma than in serum [14, 31, 32]. Levels of some cytokines can be different between matched specimens of plasma and serum collected from the same individual, and also can be affected by different anticoagulants used in plasma samples, suggesting the important roles of specimens and anticoagulants in the measurement of cytokines [33]. As a result, different ICC values of a same cytokine reported by different studies may also be caused by various specimens and anticoagulants used.

The circulating levels of cytokines are reported to be influenced by various factors including demographics (age, gender, race/ethnicity), life styles (exercise, sleep, smoking, drinking, diet and adiposity), medical treatment and circadian physiological variability $[11,18,34-$ 36]. Therefore, the inconsistency might also be partially due to the discrepancies in characteristics of the study population, and time periods across measurements [18, 31]. For instance, compared with the reproducibility of 


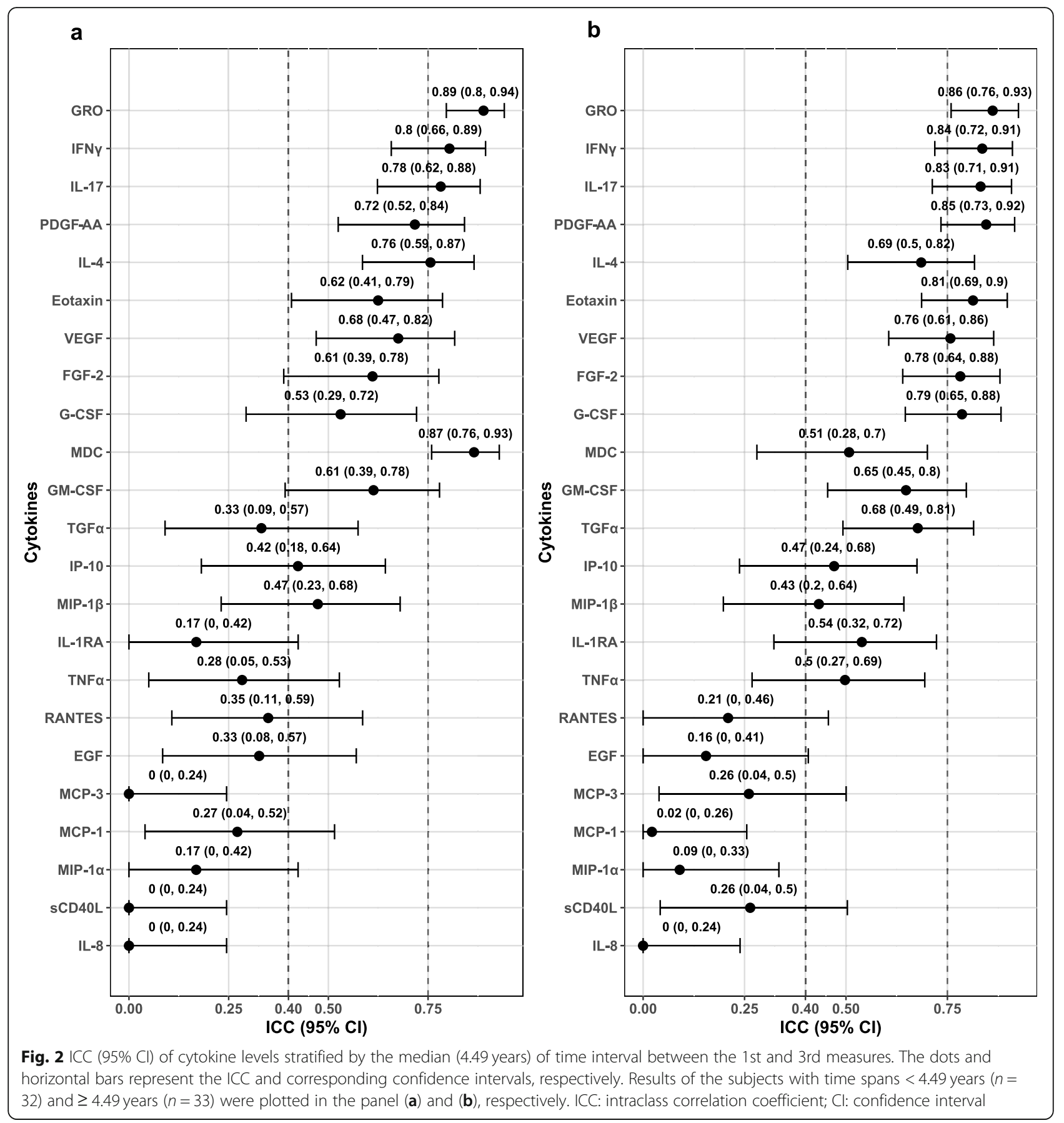

IL-4 $(\mathrm{ICC}=0.76)$ in our study, similar results were observed among the healthy women from Sweden $(\mathrm{ICC}=$ 0.70) [12] and USA (ICC =0.92) [11], but lower ICC values $(<0.40)$ were reported in populations from other areas $[17,18]$. We found an excellent reproducibility of IFNY $(\mathrm{ICC}=0.82)$ in the elderly subjects. In contract, lower values of ICC for IFNY (about 0.50) have been obtained from the younger populations for which the mean age was less than 60 years $[17,18,37]$. Data from laboratory studies indicate that the secretion of IFN $\gamma$, a pro- inflammatory cytokine mainly produced by the type $1 \mathrm{~T}$ cells, and the IFN $\gamma$ inducible inflammation cascade are increased with aging $[38,39]$. Thus, inconsistent reproducibility of IFNY between current and previous studies was supported by the aging effects.

An assessment of the long-term reproducibility of biomarkers is essential to explore the associations between biomarkers and diseases with long etiological windows, such as the neurodegenerative disease, cardiometabolic disease, or cancer. Majority of the previous studies 
evaluating the reproducibility of cytokines had used samples spanning a shorter period of time, such as 14 days [17], several months [14, 15, 18, 19, 21], $1 \sim 2$ years [16], or $2 \sim 3$ years $[11,12,37]$. The current study has a median time span of 4.49 (range $=2.86$ to 15.26 ) years between the first and the last samples, probably fitting better the goal of evaluating the long-term reproducibility of cytokines. Only two previous studies covered similar (over 5 years) [13] or markedly longer ( median $=18.3$ years) [30] periods of time. However, the generalization of results in the two previous studies might be precluded due to the small sample size $(n=28)$ [13] or restriction to males [30].

With increasing time intervals, the temporal reproducibility of G-CSF kept good to excellent over 14 days $(\mathrm{ICC}=0.50)$ [17], 7 months $(\mathrm{ICC}=0.73)$ [14], $1 \sim 3$ years $(\mathrm{ICC}=0.75) \quad[12] \quad$ and $\geq 4.49$ years $\quad(\mathrm{ICC}=0.79$, the present study). Meanwhile, previous studies showed inconsistent trends of temporal reproducibility of some cytokines within a fixed population [15, 30]. The ICC of TNF $\alpha$ was reported to be diminished from 0.54 to 0.49 with increased time intervals from $0 \sim 1.9$ to $\geq 15$ years, respectively, in males whose median age was 45.6 years [30]; however, it was reported to be increased from 0.39 to 0.47 for time spans of 6 weeks and 9 months, respectively, in the combination of males and females whose mean age was 64 years [15]. In line with the ICC trends of the second study mentioned above [15], we found a lower temporal reproducibility of TNFo in subjects with time spans $<4.49$ years $(\mathrm{ICC}=0.28)$ compared with those with longer time intervals $(\geq 4.49$ years, ICC $=$ 0.50). The ICC trends over different durations may be influenced by various factors including the physiological homeostasis, storage conditions, degradation rate, preanalytical processing, and other characteristics which can influence the biomarker levels and are changeable within a subject $[30,40]$. Further studies are needed to examine the effect of duration between samples on cytokine reproducibility.

In the sensitivity analyses without undetectable values, we found similar, although slightly reduced, temporal reproducibility for most cytokines except for MCP-3 of which the ICC increased (see Additional file 1: Fig. S2). The reduction of ICC might be due to the exclusion of subjects who had consistently low or undetectable levels of analytes across different samples. On one hand, the undetectable values of cytokines mean imprecise measurements. On the other hand, the consistently undetectable levels can reflect acceptable temporal reproducibility within a subject, implying potential usefulness of these undetectable levels. For instance, subjects with consistently low or undetectable values of cytokines can be categorized into the low "exposure" group in the epidemiological studies. The impact of undetectable biomarkers was limited in our study because cytokines with inadequate detection rates $(<60 \%)$ were excluded in the primary analyses [11, 14]; and similar results were observed when additionally removing the subjects with undetectable values of cytokines (see Additional file 1: Fig. S2).

Results from previous studies demonstrated sex differences in levels of some cytokines among young adults (median age $=22$ years) [41], and in cytokine secretion responsiveness of lymphomonocytes under stress conditions [42]. We found nonsignificant differences of cytokine levels (data not shown, $p>0.10$ ) and similar reproducibility of cytokines between older males and females in this study, which might be due to the depletion of sex steroid hormones in elderly subjects. Although it has been reported that BMI is significantly correlated with levels of cytokines in cerebrospinal fluid [43], and that circadian clock is a regulator of cytokines [36], we found robust ICC of serum cytokines when additionally adjusting for BMI or time of day of blood sample collection, indicating limited influence of BMI and time of day of blood drawn on the reproducibility of cytokines in the present study.

There were several advantages in this study. Few studies have examined the elderly population previously. Our study was performed among subjects with a mean age of 77.89 years, which provided useful information for the studies focusing on the health effects of cytokines among elderly individuals. In addition, a large panel of cytokines (41 in total) were analyzed in the present study, while fewer (no more than 20) cytokines were investigated in some previous studies [13, 15-19, 21, 37]. Additionally, because subjects in this study were racially diverse and community-sourced, our findings can be widely generalized.

Some limitations should be noted. Although this study represent one of the largest study in terms of number of cytokines measured, a total of 41 cytokines may still not give a comprehensive insight into the overall inflammatory profile, which may involve more cytokines $[44,45]$. Besides, cytokine ICC could not be estimated by stratification of race due to the limited sample size. Secondly, while subjects in this study were limited to those without dementia, the influences of other diseases could not be fully excluded. However, the results were similar when adjusting for the history of hypertension, diabetes and cardiovascular diseases, suggesting limited influence of these comorbidities. Although incident diseases developed in the midst of blood collection intervals may disrupt the cytokine homeostasis and enhance the variability of cytokine levels, inducing the underestimation of cytokine ICC, it is still reasonable to use the selected cytokines with good reproducibility in the future epidemiological studies to investigate the cytokine- 
associated diseases. Thirdly, the disparity in time intervals might have induced biased estimation of ICC. To overcome this issue, the ICC was calculated with adjustments of time intervals. We also found robust results after a series analyses within subgroups with shorter intervals between samples, suggesting limited impacts of time intervals on the temporal reproducibility of selected cytokines. Fourthly, the results should be interpreted with caution for the cytokines of which the point estimation of ICC was more than the cut-off value of 0.40 but the confidence intervals included 0.40 (IP-10, MIP-1 $\beta$, IL-1RA).

\section{Conclusions}

In conclusion, the present study demonstrated a good temporal reproducibility of 15 serum cytokines (Eotaxin, VEGF, FGF-2, G-CSF, MDC, GM-CSF, TGF $\alpha$, IP-10, MIP-1 $\beta$, IL-1RA, GOP, IFN $\gamma$, IL-17, PDGF-AA, IL-4) which were measured by the multiplex technology. This suggests that a single measurement of the selected cytokines is likely to be suitable for characterizing the immune and inflammation status over a long period in the prospective epidemiological studies, especially for the elderly population.

\section{Supplementary information}

Supplementary information accompanies this paper at https://doi.org/10. 1186/s12979-020-00201-0.

Additional file 1: Table S1. Assessments on the temporal

reproducibility of circulating cytokines in previous studies. Fig. S1. Paired repeated measures correlation among different cytokines. Fig. S2. ICC ( $95 \% \mathrm{Cl}$ ) of cytokines by excluding the subjects with undetectable values.

Fig. S3. ICC ( $95 \% \mathrm{Cl})$ of cytokines when additionally adjusting for baseline comorbidities. Fig. S4. ICC (95\% CI) of cytokines when additionally adjusting for BMI categories. Fig. S5. ICC (95\% Cl) of cytokines when additionally adjusting for time of day of blood sample collection. Fig. S6. ICC (95\% Cl) of cytokines stratified by sex.

\section{Abbreviations \\ BMI: Body mass index; Cl: Confidence interval; CV: Intra-assay coefficient of variation; EGF: Epidermal growth factor; FGF-2: Fibroblast growth factor 2; Flt- 3 L: Fms-like tyrosine kinase 3 ligand; G-CSF: Granulocyte colony-stimulating factor; GM-CSF: Granulocyte macrophage colony-stimulating factor; GRO: Growth regulated oncogene; ICC: Intraclass correlation coefficient; IFNa2: Interferon alpha 2; IFNy: Interferon gamma; IL: Interleukin; IL- 1RA: Interleukin-1 receptor antagonist; IP-10: Interferon gamma-induced pro- tein 10; IQR: Interquartile range; LLOD: Lower limit of detection; MCP: Monocyte chemotactic protein; MDC: Macrophage-derived chemokine; MIP: Macrophage inflammatory proteins; PDGF: Platelet-derived growth factor; RANTES: Regulated on activation, normal T cell expressed and secreted; SD: Standard deviance; TGF: Transforming growth factor; TNF: Tumor necrosis factor; ULOD: Upper limit of detection; VEGF: Vascular endothelial growth factor; WHICAP: Washington Heights-Inwood Community Aging Project; log: Natural logarithm; sCD40L: Soluble cluster of differentiation 14 ligand}

\section{Acknowledgements}

This manuscript has been reviewed by WHICAP investigators for scientific content and consistency of data interpretation with previous WHICAP Study publications. We acknowledge the WHICAP study participants and the WHICAP research and support staff for their contributions to this study. This study was supported by the Biomarkers Core Laboratory at the Irving Institute for Clinical and Translational Research, home to Columbia University's Clinical and Translational Science Award Program hub.

\section{Authors' contributions}

Conceptualization: YG; Data acquisition: YG, NS; Data analysis: JG; Data interpretation: JG, YG; Drafting manuscript: JG; Revising manuscript: YG, NS, $\mathrm{RM}$; Obtaining funding: $Y G, R M$; Approving final version of the manuscript: all authors.

\section{Funding}

Data collection and sharing for this project was supported by the Washington Heights-Inwood Columbia Aging Project (WHICAP, P01AG007232, R01AG037212, RF1AG054023, AG042483, AG059013) funded by the National Institute on Aging (NIA) and by the National Center for Advancing Translational Sciences, National Institutes of Health, through Grant Number UL1TR001873, and Taub Institute Innovation Award.

\section{Availability of data and materials}

The data that support the findings of this study will be made available from the authors upon reasonable request and with permission of the WHICAP Study Committee.

\section{Ethics approval and consent to participate}

The study protocols were approved by the Institutional Review Board at Columbia University Medical Center. Written informed consent was provided by all the participants.

\section{Consent for publication}

Not applicable.

\section{Competing interests}

The authors declare that they have no competing interests.

\section{Author details}

${ }^{1}$ Taub Institute for Research in Alzheimer's Disease and the Aging Brain Columbia University, New York, NY, USA. ²Department of Neurology, Columbia University, New York, NY, USA. ${ }^{3}$ Gertrude H. Sergievsky Center, Columbia University, New York, NY, USA. ${ }^{4}$ Department of Epidemiology, Joseph P. Mailman School of Public Health, Columbia University, New York, NY, USA.

Received: 17 June 2020 Accepted: 29 September 2020

Published online: 13 October 2020

\section{References}

1. Commins SP, Borish L, Steinke JW. Immunologic messenger molecules: cytokines, interferons, and chemokines. J Allergy Clin Immunol. 2010;125(2 Suppl 2):S53-72.

2. Altan-Bonnet G, Mukherjee R. Cytokine-mediated communication: a quantitative appraisal of immune complexity. Nat Rev Immunol. 2019;19(4): 205-17.

3. Schett $G$, Elewaut D, McInnes IB, Dayer JM, Neurath MF. How cytokine networks fuel inflammation: toward a cytokine-based disease taxonomy. Nat Med. 2013;19(7):822-4.

4. Williams JW, Huang LH, Randolph GJ. Cytokine circuits in cardiovascular disease. Immunity. 2019;50(4):941-54.

5. Becher B, Spath S, Goverman J. Cytokine networks in neuroinflammation. Nat Rev Immunol. 2017;17(1):49-59.

6. Lambrecht BN, Hammad H, Fahy JV. The cytokines of asthma. Immunity. 2019;50(4):975-91.

7. West NR, McCuaig S, Franchini F, Powrie F. Emerging cytokine networks in colorectal cancer. Nat Rev Immunol. 2015;15(10):615-29.

8. Georgakis MK, Gill D, Rannikmae K, Traylor M, Anderson CD, Lee JM, et al. Genetically determined levels of circulating cytokines and risk of stroke. Circulation. 2019;139(2):256-68

9. Mehta RS, Chong DQ, Song M, Meyerhardt JA, Ng K, Nishihara R, et al. Association between plasma levels of macrophage inhibitory Cytokine-1 before diagnosis of colorectal Cancer and mortality. Gastroenterology. 2015; 149(3):614-22 
10. Allswede DM, Yolken RH, Buka SL, Cannon TD. Cytokine concentrations throughout pregnancy and risk for psychosis in adult offspring: a longitudinal case-control study. Lancet Psychiatry. 2020;7(3):254-61.

11. Gu Y, Zeleniuch-Jacquotte A, Linkov F, Koenig KL, Liu M, Velikokhatnaya L, et al. Reproducibility of serum cytokines and growth factors. Cytokine. 2009; 45(1):44-9.

12. Clendenen TV, Arslan AA, Lokshin AE, Idahl A, Hallmans G, Koenig KL, et al. Temporal reliability of cytokines and growth factors in EDTA plasma. BMC Res Notes. 2010;3:302.

13. Hofmann JN, Yu K, Bagni RK, Lan Q, Rothman N, Purdue MP. Intra-individual variability over time in serum cytokine levels among participants in the prostate, lung, colorectal, and ovarian cancer screening trial. Cytokine. 2011; 56(2):145-8.

14. Belzeaux R, Lefebvre MN, Lazzari A, Le Carpentier T, Consoloni JL, Zendjidjian $\mathrm{X}$, et al. How to: measuring blood cytokines in biological psychiatry using commercially available multiplex immunoassays. Psychoneuroendocrinology. 2017;75:72-82.

15. Todd J, Simpson P, Estis J, Torres $V$, Wub AH. Reference range and short- and long-term biological variation of interleukin (IL)-6, IL-17A and tissue necrosis factor-alpha using high sensitivity assays. Cytokine. 2013; 64(3):660-5.

16. Lee SA, Kallianpur A, Xiang YB, Wen W, Cai Q, Liu D, et al. Intra-individual variation of plasma adipokine levels and utility of single measurement of these biomarkers in population-based studies. Cancer Epidemiol Biomark Prev. 2007;16(11):2464-70.

17. Yeh TC, Chu HT, Tsai CK, Chang HA, Yang FC, Huang SY, et al. Distinct inflammation biomarkers in healthy individuals and patients with schizophrenia: a reliability testing of multiplex cytokine immunoassay by bland-Altman analysis. Psychiatry Investig. 2019;16(8):607-14.

18. Koelman L, Pivovarova-Ramich O, Pfeiffer AFH, Grune T, Aleksandrova K. Cytokines for evaluation of chronic inflammatory status in ageing research: reliability and phenotypic characterisation. Immun Ageing. 2019;16:11.

19. Buxton MA, Meraz-Cruz N, Sanchez BN, Gronlund CJ, Foxman B, VadilloOrtega $\mathrm{F}$, et al. Air pollution and inflammation: findings from concurrent repeated measures of systemic and reproductive tract cytokines during term pregnancy in Mexico City. Sci Total Environ. 2019;681:235-41.

20. Biancotto A, Wank A, Perl S, Cook W, Olnes MJ, Dagur PK, et al. Baseline levels and temporal stability of 27 multiplexed serum cytokine concentrations in healthy subjects. PLoS One. 2013;8(12):e76091.

21. Navarro SL, Brasky TM, Schwarz Y, Song $X$, Wang CY, Kristal AR, et al. Reliability of serum biomarkers of inflammation from repeated measures in healthy individuals. Cancer Epidemiol Biomark Prev. 2012;21(7):1167-70.

22. Pinti M, Appay V, Campisi J, Frasca D, Fulop T, Sauce D, et al. Aging of the immune system: focus on inflammation and vaccination. Eur J Immunol. 2016;46(10):2286-301.

23. Ferrucci L, Fabbri E. Inflammageing: chronic inflammation in ageing, cardiovascular disease, and frailty. Nat Rev Cardiol. 2018;15(9):505-22.

24. Manly JJ, Bell-McGinty S, Tang MX, Schupf N, Stern Y, Mayeux R. Implementing diagnostic criteria and estimating frequency of mild cognitive impairment in an urban community. Arch Neurol. 2005;62(11): 1739-46.

25. Kulick ER, Elkind MSV, Boehme AK, Joyce NR, Schupf N, Kaufman JD, et al. Long-term exposure to ambient air pollution, APOE-epsilon4 status, and cognitive decline in a cohort of older adults in northern Manhattan. Environ Int. 2020;136:105440

26. The Food and Drug Administration. Bioanalytical Method Validation Guidance for Industry. https://www.fda.gov/regulatory-information/searchfda-guidance-documents/bioanalytical-method-validation-guidance-industry [accessed on Sep 1, 2020]. 2018.

27. Bakdash JZ, Marusich LR. Repeated measures correlation. Front Psychol. 2017:8:456.

28. Bates D, Machler M, Bolker BM, Walker SC. Fitting linear mixed-effects models using Ime4. J Stat Softw. 2015;67(1):1-48

29. Pleil JD, Wallace MAG, Stiegel MA, Funk WE. Human biomarker interpretation: the importance of intra-class correlation coefficients (ICC) and their calculations based on mixed models, ANOVA, and variance estimates. J Toxicol Environ Health B Crit Rev. 2018;21(3):161-80.

30. McKay HS, Margolick JB, Martinez-Maza O, Lopez J, Phair J, Rappocciolo G, et al. Multiplex assay reliability and long-term intra-individual variation of serologic inflammatory biomarkers. Cytokine. 2017:90:185-92.
31. Chaturvedi AK, Kemp TJ, Pfeiffer RM, Biancotto A, Williams M, Munuo S, et al. Evaluation of multiplexed cytokine and inflammation marker measurements: a methodologic study. Cancer Epidemiol Biomark Prev. 2011;20(9):1902-11.

32. Altara R, Manca M, Hermans KC, Daskalopoulos EP, Brunner-La Rocca HP, Hermans RJ, et al. Diurnal rhythms of serum and plasma cytokine profiles in healthy elderly individuals assessed using membrane based multiplexed immunoassay. J Transl Med. 2015;13:129.

33. Biancotto A, Feng $X$, Langweiler M, Young NS, McCoy JP. Effect of anticoagulants on multiplexed measurement of cytokine/chemokines in healthy subjects. Cytokine. 2012;60(2):438-46.

34. O'Connor MF, Irwin MR. Links between behavioral factors and inflammation. Clin Pharmacol Ther. 2010;87(4):479-82.

35. Clendenen TV, Koenig KL, Arslan AA, Lukanova A, Berrino F, Gu Y, et al. Factors associated with inflammation markers, a cross-sectional analysis. Cytokine. 2011:56(3):769-78.

36. Nakao A. Temporal regulation of cytokines by the circadian clock. J Immunol Res. 2014;2014:614529.

37. Epstein MM, Breen EC, Magpantay L, Detels R, Lepone L, Penugonda S, et al. Temporal stability of serum concentrations of cytokines and soluble receptors measured across two years in low-risk HIV-seronegative men. Cancer Epidemiol Biomark Prev. 2013;22(11):2009-15.

38. Yen CJ, Lin SL, Huang KT, Lin RH. Age-associated changes in interferongamma and interleukin-4 secretion by purified human CD4+ and CD8+ T cells. J Biomed Sci. 2000;7(4):317-21.

39. Oxenkrug G. Interferon-gamma - inducible inflammation: contribution to aging and aging-associated psychiatric disorders. Aging Dis. 2011;2(6): 474-86.

40. Aziz N, Detels R, Quint JJ, Li Q, Gjertson D, Butch AW. Stability of cytokines, chemokines and soluble activation markers in unprocessed blood stored under different conditions. Cytokine. 2016;84:17-24.

41. Bernardi S, Toffoli B, Tonon F, Francica M, Campagnolo E, Ferretti T, et al. Sex Differences in Proatherogenic Cytokine Levels. Int J Mol Sci. 2020;21(11).

42. Da Pozzo E, Giacomelli C, Cavallini C, Martini C. Cytokine secretion responsiveness of lymphomonocytes following cortisol cell exposure: sex differences. PLoS One. 2018;13(7):e0200924.

43. Larsson A, Carlsson L, Lind AL, Gordh T, Bodolea C, Kamali-Moghaddam M, et al. The body mass index (BMI) is significantly correlated with levels of cytokines and chemokines in cerebrospinal fluid. Cytokine. 2015;76(2):514-8.

44. O'Shea JJ, Murray PJ. Cytokine signaling modules in inflammatory responses. Immunity. 2008;28(4):477-87.

45. Friedrich $M$, Pohin M, Powrie F. Cytokine networks in the pathophysiology of inflammatory bowel disease. Immunity. 2019;50(4):992-1006.

\section{Publisher's Note}

Springer Nature remains neutral with regard to jurisdictional claims in published maps and institutional affiliations.

Ready to submit your research? Choose BMC and benefit from:

- fast, convenient online submission

- thorough peer review by experienced researchers in your field

- rapid publication on acceptance

- support for research data, including large and complex data types

- gold Open Access which fosters wider collaboration and increased citations

- maximum visibility for your research: over $100 \mathrm{M}$ website views per year

At $\mathrm{BMC}$, research is always in progress.

Learn more biomedcentral.com/submissions 